hende Uberprüfung der Angemessenheit des Totalitarismusbegriffs für die Charakterisierung von Herrschaftsstrukturen aber wurde nicht geleistet.

Der systemtheoretisch orientierte $Z$ weig des Comparative Government geht von der prinzipiellen Vergleichbarkeit aller Gesellschaften und politischen Systeme aus. Man nimmt bestimme Funktionen des politischen Systems in allen hochindustrialisierten Gesellschaften an und untersucht Interaktionen des politischen Systems mit anderen Subsystemen. Dabei liegt das Hauptinteresse nicht auf den formalen sondern den faktischen Entscheidungs- und Legitimationsprozessen. Das Modell interagierender und kommunizierender Subsysteme soll Fragestellungen und Hypothesen formulieren helfen, die empirisch zu überprüfen sind.

Von dieser Richtung wurde das Totalitarismuskonzepr einer vielfältigen Kritik unterzogen. Einige Punkte sollen referiert werden ${ }^{b}$ : r. Das Totalitarismuskonzept impliziert eine Wertung. Es ist vorwiegend nicht empirisch sondern ideologisch orientiert. 2. Der Idealtypus kann einer empirischen Kontrolle im strengen Sinne nicht unterworfen werden. $E_{r}$ hat de facto eine verengte Blickrichtung in der Analyse der unter ihn subsummierten Staaten gefördert. "Totalitäre» Systeme wurden primär unter dem Aspekt des outputs (»pluralistische« unter dem des inputs) analysiert. 3. Das Begriffspaar Totalitarismus - Pluralismus ist auf einen amorphen Machtbegriff bezogen. Dieser ist - weil nicht quantifizierbar für vergleichende Analysen ungeeignet.

Systemtheoretisch angeleitete empirische Untersuchungen - etwa zum Problem der Interessengruppen in der Sowjetunion oder zu Fragen der Kommunikationskanäle und der Legitimationsbeschaffung der KPdSU - konnten den Totalitarismusbegriff als zu grob kritisieren und mehr und mehr durchlöchern. ${ }^{7}$

Melanie Unterseher-Jabn

\title{
Juradat kämpft gegen die Informationslawine
}

"Aufbau, Speicherung und Benurzung umfassender juristischer Datenbanken sollten der Kontrolle unabhängiger Gremien unterstellt werden, um von vornherein den Gefahren der Einseitigkeit, der Interessengebundenheit und einer etwaigen Monopolstellung zu begegnen. «1

${ }^{6}$ Vgl. u. a. K. v. Beyme, Gesellschaftliche Organisationen und Interessenpluralismus in der Sowjetunion, in: R. Löwenthal/B. Meissner (Hrg.), Sowjetische Innenpolitik, Stuttgart 1968, S. 39 ff., R. Burrows, Totalitarism - The Revised Standard Version, in: World Politics 1968/69, S. $272 ;$ P. Ch. Ludz, Parteielite im Wandel, Köln 1968.

; Vgl. u. a. M. Lodge, Soviet Elite Participatory Attitudes in the Post-Stalin Period, in: American Political Science Review :968, S. 827 ff.; G. Skilling, Interest Groups and Communist Politics, in: World Politics $x 965 / 66$, S. 435 ff.; M. P. Gehlen/M. McBridge, The Soviet Central Committec: An Elite Analysis in: American Political Science Review r 968, S. I232 ff.

1 Ständige Deputation des Deutschen Juristentags in Mainz 1970. 
$\mathrm{Daß}$ die in dieser Forderung liegende Skepsis gegenüber der Errichtung von Datenbanken leider allzu berechtigt ist, soll am Beispiel der privaten Datenbank Juradat, West-Berlin, aufgezeigt werden.

Man muß sich mir der bereits im Juli 1969 gegründeten Firma Juradat befassen, weil ihre Auswirkungen auf die deursche Gerichtsverfassung nicht zu unterschärzen sind. Der richterliche Entscheidungsprozeß wird, wenn die Firma ihren Dienstleistungsbetrieb aufgenommen hat, plötzlich durch einen starken Informationsfluß von einseitig in den Dienst der Anwälte gestellten Obergerichtsentscheidungen beeinflußr. Der Richter, dem der Zeitdrudk ohnehin schon eine oft nicht mehr zu verantwortende Straffung und Abkürzung des Verfahrens gebietet, muß sich jetzt noch mit einer Vielzahl von weiteren Entscheidungen auseinanderserzen, die ihm die Parteien als Argumente vorlegen. Er erhält Rechtssatzinformationen in Form von Obergerichtsentscheidungen, er braucht aber auch Hilfsinformationen, besonders aus der Rechtswissenschaft, sowie weitere ähnlich gelagerte Obergerichtsurteile, um sich mit ihnen auseinandersezzen zu können. Rechtswissenschaftliche Informationen liefert Juradat aber gerade nicht. Während die Anwälte den Richter mit elektronisch gefundener Information bombardieren, muß er nach alter, nicht bewährter Art in der Gerichtsbibliothek nach Aufsätzen, Monographien und anderen Obergerichtsurteilen fahnden. Der Anwalt wird nur die ihm günstigsten Urteile vorlegen, der Richter ist also letztlich auf eine Anfrage bei Juradat angewiesen. Er begibr sich damit in den gleichen Teufelskreis der Informationsbeschaffung durch Juradat, der bestimmt ist durch problematische Einspeicherung, nicht erkennbare Auswahlkriterien, fehlerhafte Auswahl, unvollständige Deskriptoren etc., dem das vom Anwalt ausgewählte Urteil schon unterlag. Uber den $Z$ wang zur Informationsbeschaffung wird Juradat also zur Pflichrinstanz in der deutschen Gerichtsverfassung.

II

Von erheblicher Bedeutung für die Arbeitsweise und damit auch für die Möglichkeit der Kontrolle ist die Organisationsform der Firma Juradat GmbH \& Co. Betriebs-KG, die den Gesellschaftern die Möglichkeit gibt, die gesellschaftsrechtlichen Verhältnisse in der Satzung frei auszugestalten und die die ungezwungene institutionalisierte unöffentliche Kooperation von öffentlicher Bürokratie und Privatkapital erleichtert. ${ }^{2}$ Außerdem kann in dieser Organisationsform mit den eingesparten Steuern und den staatlichen Subventionen (die es in West-Berlin immer gibt) schnell Geld gemacht werden.

Wird auch hinsichtlich der Geldgeber - unter ihnen ein Möbelhändler, ein Zahnarzt, Rechtsanwälte - offiziell geheimnisvolles Schweigen gewahrt, so spricht doch außer der Organisationsform der Firma einiges mehr - und noch beredter für die innere Abhängigkeir von kapitalistischen Interessen:

Der geschäftsführende Gesellschafter Ing. J. Wander, ehemals Fuhrparkleiter einer Kiesfirma, ist Direktor einer "NEWA-Fachschule für Organisation und Datenverarbeitung « und Vorsitzender des Vorstands des Vereins deutscher Organisationstechniker (VDOT). In dem in Bau befindlichen »Haus der Organisation « in West-Berlin sollen neben Juradat auch Schulungsräume für interessierte

2 Lukas Niemann, KJ x 970 , S. 75. 
Organisationen und Wirtschaftsunternehmen, Ausstellungsräume sowie Räume für die NEWA-Fachschule und den VDOT untergebracht werden.

Wander sagt unmißverständlich: "Diese Kombination wird die uneingeschränkte Zustimmung der Wirtschaft finden.\& Zustimmung der juristischen Praktiker oder gar der Wissenschaft zu dieser typischen Verquidkung erhofft und erwartet man erst gar nicht. Fazit: Offentliche Kontrolle besteht nicht, kann nicht bestehen; wenn einer kontrolliert, dann die Industrie, die wirtschaftlichen Interessen, die Gesellschafter, die Rendite sehen wollen. Offentliche Kontrolle aber ist hier unabdingbar. Die Informationen, die der Jurist bei seiner Tätigkeit aufnimme, werden nicht nur im Hinblidk auf die von ihm zu treffende "Entscheidung "ausgewählt, sie beeinflussen auch ihrerseits eben diese Entscheidung. Die Entscheidung als Ergebnis eines Informationsvorganges wird durch vorher gegebene Zielsetzungen und Präferenzregeln, nach denen die Ausscheidung von für unwesentlich gehaltener Information erfolgt, geprägt. Einrichtungen, die der Sammlung, Ordnung und Erschließung von Information dienen wollen, müssen also daraufhin überprüft werden, inwieweit ihr Vorgehen möglichst umfassend, durchschaubar und vielseitig auswertbar ist, damit mögliche Entscheidungsalternativen nicht von vornherein verbaut werden, ohne daß dies dem die Entscheidung treffenden Juristen überhaupt bewußt wäre.

In diesem Zusammenhang wird auch die Problematik der Speicherung abweichender Rechtsmeinungen aktuell. Es besteht die Gefahr der beabsichtigten oder unbeabsichtigten Manipulierung der Chancen einer Änderung der Rechtsprechung. Diese Problematik will man bei Juradat jedoch nicht sehen.

Die Gefahren, die sich bei einer juristischen Datenbank und insbesondere bei einer privarwirtschaftlich organisierten wie Juradat ergeben, werden dann potenziert wirksam werden, wenn Juradat eine Monopolstellung bekommen wird. Dann nämlich kann die bislang fehlende Kontrolle und die Gefahr des Ausschlusses von Teilnehmern erhebliche Auswirkungen insbesondere auf die Rechtspraxis haben.

Für den Bereich der Datenbanken sind zweifellos die technischen Notwendigkeiten einer Konzentration und Monopolisierung vorhanden, da die Kosten einer EDV-Anlage, die Personalkosten und der begrenzte Kreis der Abnehmer von Informationen kaum existenzfähige Konkurrenzunternehmen zuläßt. Die $\mathrm{Ge}-$ samtkosten für Juradat werden zunächst mit I 2 Millionen DM veranschlagt.

Dem entspricht es, daß sich in Westdeutschland nur zwei weitere Datenbanken im Aufbau befinden - und weitere nicht geplant sind:

- die Nürnberger Firma DATEV, die sich des Steuerrechts annimmt,

- die Bundesdatenbank, deren Aufbau angeblich erfreulich gründlich vorbereitet werden soll und deshalb noch einige Jahre in Anspruch nehmen wird.

Somit wird ab 197 I Juradat die einzige Datenbank für den Bereich der BRD sein, die Gerichtsurteile liefern kann. Hat die Firma jedoch erst einmal eine Monopolstellung erreicht, so wird es selbst mit einer zufriedenstellenden Bundesdatenbank sehr schwer sein, daran in absehbarer Zeit etwas zu ändern. Eine Monopolisierung der Informationsvermitrlung in der Hand von Juradat bedeutet aber, daß die Gesellschafter dieser Firma den Kreis der Benutzer bestimmen und maßgeblichen Einfluß auf sie nehmen können.

r. Zum einen besteht die Gefahr, daß Juradat einzelne Kunden nicht zuläßr, sei 
es einfach auf Grund der ihr als Privatunternehmen unbenommenen Vertragsfreiheit, sei es durch Tarifgestaltung. Zwar kann der Preis nicht so hoch angesetzt werden, daß nur ein sehr kleiner Kreis sich diesen Informationsluxus leisten kann, andererseits ist Juradat gezwungen, seinen Gesellschaftern einen Profit zu erwirsschaften. Nimme man an, daß sich die Preisgestaltung nicht grundlegend von der anderer Datenbanken unterscheider, so kann man am Beispiel der Tarife des "US Law Research Service leicht erkennen, daß eine Benutzung für Studenten, Referendare und auch für die Universitäten aus finanziellen Gründen ausgeschlossen isr (7S \$ Pauschale pro Rechtsgebier, ro \$ für 4 Angaben, 2 S für je zwei Zeilen). Eine Benachreiligung gegenüber ihren besser gestellten Kollegen ist demnach auch für diejenigen Rechrsanwälte zu befürchten, die sich regelmäßige Juradat-Informationen finanziell nicht leisten können. Datenbankinformation - und damit einen erheblichen Vorteil vor dem Prozeßgegner - können sich somit nur die Reichen, bzw. deren Anwälte leisten. Die schon beute offensichtliche Benachteiligung der ärmeren Partei etwa im Zivilprozeß wird bedeutend verstärkt.

Der um Objektivität bemühte Richter kann diesen einseitigen Informationsvorsprung nicht korrigieren ohne seinerseits sich von einer Darenbank informieren zu lassen. Doch haben die Gerichte dafür kein Geld.

2. Daß auch eine zweite Gruppe von den Juradat-Informationen ausgeschlossen werden soll, ergibt sich unter vielen anderen sicheren Indizien aus einer Mitteilung von Ing. Wander. ${ }^{3}$ Die Leistungen von Juradat will er »für Rechtsanwälte, Rechtsabteilungen von Unternehmen, Behörden, Gerichte, Organisationen der Gesellschaft und sonst interessierte Kreise« zur Verfügung stellen. Man muß vermuten, daß die Universitäten, daß die gesamte Rechtswissenschaft nicht einmal unter die "sonstigen Kreise " fallen werden. Dies nicht etwa, weil die Rechtswissenschaft kein Interesse an der Dokumentation des Rechts hätte, sondern allein schon dadurch, daß von vornherein so gearbeitet wird, daß wissenschaftlich arbeitende Juristen kein Interesse an dem haben können - und offenbar auch nicht haben sollen - was ihnen Juradat anzubieten hat.

IV

Die Firma Juradat nahm im November 1969 in West-Berlin ihre Arbeit auf: in einen Großcomputer (Univac 418/III) werden die Gerichtsentscheidungen bzw. deren Leitsätze gespeichert, die bisher bearbeitet worden sind. Ab Frühjahr 1971 sollen sie den »interessierten Kreisen « verkauft werden. $Z$ war ist im Handelsregister als Gegenstand des Unternehmens u. a. angegeben: "Die Auswertung von Daten aller Art gegen Entgelt... sowie die Durchführung von wissenschaftlichen Arbeiten auf juristischem Gebiet. *

Um die Aufgaben ausreichend bewältigen zu können »bedarf es eines größeren Forschungsaufwandes. Um diesen Anforderungen gerecht zu werden, hat Juradat einen wissenschaftlichen Beirat bestellt. Diesem Beirat gehören nur Persönlichkeiten an, die »besondere Kennenisse, Verdienste und Erfahrungen auf dem Tätigkeitsgebiet der Gesellschaft haben."

Diese so Befähigten und ihr jeweiliger Arbeitsbereich sind:

Blei (Berlin), StGB, StPO;

Henrich (Regensburg), Zivilrecht;

${ }^{3}$ Pressekonferenz der Juradat am 4. 3. 1970 in West-Berlin. 
Herzog (Speyer), Staats- und Verwaltungsrecht;

Rüthers (Berlin), Arbeitsrectr;

Schumann (Regensburg), ZPO;

Sieg (Berlin), Versicherungsrecht;

Hans Wolfgang Treppe (Angehöriger der Organisationsabteilung der Berliner Justizverwaltung).

Dieser Beirat besteht nur aus Juristen. Keiner von ihnen ist durch Forschungstätigkeit auf den Gebieten der Rechtsinformatik, Systemtheorie, juristischer Sprachanalyse, Dokumentation, oder auch nur juristischer Logik hervorgetreten. Keine der Persönlichkeiren verfügr über die gepriesenen »besonderen Kenntnisse, Verdienste und Erfahrungen auf diesem Gebiet«. Die Vermutung, daß die Beiratsmitglieder mehr am Honorar als an ihrer wissenschaftlichen Mitarbeit interessiert sind, dürfte kaum mir konkreten Angaben widerlegt werden können. Selbst wenn man Prof. Bleis Angabe, daß er monatlich einen »fünfstelligen Betrag " bezieht, als Prahlerei wertet, so dürfte es sich doch um beträchtliche Summen handeln.

$\mathrm{Daß}$ die Geldgeber einen bestimmenden Einfluß auf Juradat ausïben, wurde bereits betont; inwieweit er sich praktisch auswirkt, in Verbindung mit praktischer Unerfahrenheit, ja Unfähigkeit zu wissenschaftlich einwandfreier Arbeit, zeigt sich an Auswahl und Speicherung sehr deutlich.

Von einem juristischen Informationssystem muß erwartet werden, daß es das geltende Recht, die Rechtssprechung und die juristische Literatur erschließt, wobei Querverbindungen $z$ wischen allen Informationsquellen möglich sein sollten. Der Aufbau eines solchen umfassenden Systems kann zwangsläufig nur schrittweise vollzogen werden.

Es war deshalb für Juradat naheliegend, mit der Bearbeitung einer dieser Informationsquellen zu beginnen und dabei die nötigen Erfahrungen zu sammeln. Doch ist inzwischen klar geworden, daß es bei der Dokumentierung einer dieser Quellen (Rechtsprechung) bleiben wird, ein umfassendes Informationssystem also nicht (mehr) angestrebt wird. Daher stellt Juradats Beschränkung auf die Dokumentation von Urteilen eine bewußte, gewollte und eingestandene Mißachtung (Motto: Das interessiert doch keinen Praktiker!) und somit eine endgültige Ausschaltung der Rechtswissenschaft aus diesem Bereich dar. Deutlich zeigt sich dies auch daran, daß bei der *Bearbeitung " alle Hinweise des Original-Urteils auf die Rechtslehre, jede Auseinandersetzung mit außergerichtlichen Meinungen kurzerhand weggestrichen werden.

Nicht eingespeichert und somit als für die Praxis als bedeurungslos behandelt werden:

- alle vor 1950 liegenden Entscheidungen

- alle unterhalb der OLG-Ebene ergehenden Urteile und

- alle Entscheidungen, die altes Recht anwenden.

Mit überraschender Großzügigkeit wird also "beschlossen «, daß RG-Urteile heute niemanden mehr interessieren; wie selbstverständlich geht man davon aus, $\mathrm{da}$ Untergerichtsurteile prinzipiell keine Bedeutung außerhalb ihres eigenen Verfahrens haben (denn man will ja nicht helfen, besser Recht zu sprechen, sondern Urteile "revisionssicher « zu machen) und mit Leichtigkeit vermag man zu erkennen, daß ein gerichtlich behandeltes Problem schon deshalb problemlos wurde, weil der entsprechende Paragraph geändert, das Gesetz neu erlassen oder bloß neu bekanntgemacht wurde.

Es wird also eifrig ausgewählt, eigentlich mehr weggelassen als ausgewählt denn man hat ja nur eine begrenzte Speicherkapazität. 
Zwar beruht Information immer aus einer Auswahl, doch da Auswahl zugleich eine Aus-wertung ist, sind Wertvorstellungen, spezielle Interessenlagen, Ideologien wirksam. Die Informationsbeschaffung kann den Benutzer also in irgendeine Ideologie einspannen. Das geschieht oft unmerklich und nur sehr schwach, manchmal aber auch unmerklich und sehr stark.

Bis es einer juristischen Information gelingt, Eingang in den Juradat-Computer zu finden, sind so viele Auswahlprozesse - angefangen von der das Urteil einsendenden und kürzenden Gerichtsstelle über die auswählende und kürzende Zeirschriften-Redaktion bis zum auswählenden und kürzenden Juradat-Arbeiter - über dies Urteil hinweggegangen, daß es letztlich eine Frage des Zufalls ist, ob es für dokumentationswürdig erklärt wird.

Ist es einer Entscheidung endlich gelungen, Aufnahme in den Kreis derer zu finden, mit denen sich vermutlich Geld verdienen läßt (juristische Information als Ware mit steuerbarem Angebor und beeinflußbarer Nachfrage), so wird sie sich kaum mehr wiedererkennen: Sie ist auf ihren wwesentlichen Kern " zurechtgestutzt. In den Anweisungen für die Bearbeiter wird hierzu folgendes ausgeführt: "Die Entscheidungen sind durch sinnvolle Streichungen auf ihren wesentlichen Gehalt zurückzuführen. Der vom Bearbeiter (Referendar) für speichernswert gehaltene Text ist durch... Klammern kenntlich zu machen. Es ist darauf zu achten, daß die kostbare Speicherkapazität nicht durch die Aufnahme überflüssiger Textabschnitte belastet wird. " $Z$ war soll durch diese Sparsamkeit »das Verständnis des verbleibenden Entscheidungstextes« nicht leiden, doch »ist folgendes einzuhalten:

a) Für das Verständnis der Entscheidung Unwesentliches wird weggelassen.

b) Der Sachverhalt (Tatbestand) wird nur mit aufgenommen, falls und soweit er zum Verständnis der Gründe unerläßlich ist.

c) Zitate in der Entscheidung: Literatur- und Kommentarzitate... weglassen ... Unveröffentlichte Entscheidungen wegstreichen.

d) Obsolete oder ausdiskutierte Rechtsfragen sind wegzulassen oder nur kurz wiederzugeben (z. B.: »Verkehrszeichen sind Verwaitungsakte ")《.

Dies macht bereits stutzig; schlimmer noch ist die Wirklichkeit: etwa 17 Referendare, deren einzige Qualifikation für diese Aufgabe die Kenntnis dieser Anweisungen ist, streichen weg, was immer gerade sie für streichenswert halten. Immer die »kostbare Speicherkapazität« vor Augen, durchforsten sie BGH und BVerfGEntscheidungen nach »überflüssigen Textabschnitten $\times$. Wer kann mit Sicherheir entscheiden, was das »Unwesentiiche" einer Entscheidung ist? Sind lediglich angeschnittene Fragen, dahingestellt Gelassenes, sind obiter dicta wirklich »unwesentlich«? Der Sachverhalt ist zum Verständnis der Gründe immer erforderlich!

Unsere Kritik an der Arbeitsweise richter sich somit nicht nur dagegen, daß ausgewählt und gekürzt wird, sondern auch dagegen, wie dies gemacht wird. Mindestvoraussetzung für eine Dokumentation des Rechts ist ein gut durchdachtes, wissenschaftlich fundiertes, konsequent und einheitlich angewandtes detailliertes System der Auswahl-Kriterien.

Doch existiert dies bei Juradat auch nicht in Ansätzen. I7 Referendare haben I7 "Systeme", deren Grundregel lauter: geringer Denkaufwand - was angesichts der Tatsache, daß man sie nicht über die Bedeutung der Sache, den technischen und rechtspolitischen Hintergrund aufgeklärt hat, nicht verwundern kann. Erfahrungen in anderen Ländern haben übrigens gezeigt, daß selbst qualifizierte Bearbeiter dieselbe Entscheidung, wenn sie ihnen versehentlich mehrfach vorgelegt wurde, jedesmal erheblich anders bearbeiteten!

Die bisherige Kritik gilt im wesentlichen auch für die Arbeit, die zur Wiederauf- 
findung der eingespeicherten Dokumente geleistet werden muß: die Kennzeichnung eines jeden Dokuments mit den charakteristischen Schlüsselwörtern, Deskriptoren, für die Erstellung des zentralen Thesaurus sowie für das Verfahren des Wiederauffindens der eingespeicherten Informationen. Auf die Einzelheiten, die diese Behauptung stützen, kann hier nicht näher eingegangen werden. Hierfür wäre eine Schilderung der technischen, sprachwissenschaftlichen und informationstheoretischen Voraussetzungen für die Informationswiedergewinnung und über die Funktion und Aufbau des Thesaurus erforderlich. ${ }^{4}$

In den USA hat man für die Erforschung solcher Systeme viele Jahre benötigt. Hier davon zu sprechen, daß sich »die bis ins Detail gehende Forschungsarbeit... über mehrere Monate hinziehen « werde, zeugt von einem bewunderungswürdigen Optimismus bzw. bedauerlichen Dilettantismus der Firma Juradat. Weiterhin ist es nicht sinnvoll, die Fachgebietssysteme allein von Juristen ausarbeiten zu lassen, und erst dann diese Systeme mit der Datenverarbeitungsanlage abzustimmen, wie dies in Berlin geschieht.

\section{V}

Die Darstellung der organisations- und dokumentationstechnischen Mängel ist nur dann sinnvoll, wenn immer wieder über die damit verbundenen praktischen Probleme hinaus auf die rechtspolitischen Konsequenzen hingewiesen wird. Die Gefahr des Entstehens und Verfestigens einer neuen Verfügungsmachr über für die Gesellschaft notwendige Information ohne zureichende öffentliche Kontrolle ist zu groß, als daß man die wirtschaftliche Basis und die ökonomischen Interessen eines solchen Unternehmens, das so leichtfertig mit der Ware "Information « hantiert, akzeptieren könnte. Das scheinbar liberale Argument, die Firma Juradat sei doch ein Unternehmen wie jedes andere auch, unterscheide sich z. B. kaum von einem juristischen Fachverlag, verkennt die Problematik: Juradat will später vornehmlich brandneue Urteile anbieten, die in den juristischen Zeitschriften noch nicht veröffentlicht sind; weiterhin wird ein mehr oder weniger starker $Z$ wang zum Mithalten auf den Prozeßgegner ausgeübt. Dabei ist zu befürchten, daß die Obergerichte sich bereit erklären könnten, die von ihnen an Juradat gelieferten Urteil(sausschnirt)e für einige Zeit, $1 / 2$ Jahr oder länger, nicht an Fachzeirschriften weiterzugeben. Die Benutzer werden gezwungen sein, sich blindlings auf die Richtigkeit d. h. auf die Vollständigkeit der gelieferten Urteile zu verlassen, und damit auf die Gewissenhaftigkeit und Fähigkeit der Bearbeiter. Was aber, wenn Informationen, die in eine bestimmte (politische) Richtung tendieren, generell für einige Zeit oder ganz gesperrt oder nur mentschärft" werden?

Wie oben gezeigt, wird die Rechtswissenschaft (im formalen Sinne) von Juradat ignoriert - gründlich und folgenreich -, wenn auch mit einer Einschränkung: ignoriert wird sie als Kunde, als Benutzer dieser privaten Datenbank, willkommen ist sie dagegen als Kräftereservoir. Wird einerseits so gearbeitet, daß der Einfluß der Wissenschaft auf die Meinungsbildung zurückgehen muß, so wird andererseits die wissenschaftliche Forschung ausgebeutet, bis sie nicht mehr benörige wird.

\footnotetext{
4 Insoweit verweisen wir auf die Ausführungen in unserer Broschüre $>$ Die Informarionsmacher. Zur Fragwürdigkeit einer privaten Datenbank für Rechtsdokumentation - die Firma JURADAT \&, hrsg. von der Arbeitsgruppe $\gg E D V$ und Rechta im FB Rechtswissenschaft an der FUB, 1970, \& Berlin 33, Van-t Hoffstr. 8, sowie auf die Darstellung der möglichen Verfahren und ihrer Probleme bei Haft, Elektronische Datenverarbeitung im Recht, 1970, S. Irs ff.
} 
Zunächst einmal braucht man sie noch:

Technische, systemtheoretische, juristische u. a. Probleme treten laufend auf; bis jetzt hofft man wohl großenteils, daß man sie ausklammern, sie umgehen könnte; doch wenn sie dringender werden, wird man sich zu ihrer Lösung vornehmlich der Universitäten bedienen.

Weiter wird man billige juristische Fachkräfte anheuern - Studenten, Assistenten, Referendare, Doktoranden. Die Beirats-Professoren werden Forschungsaufträge an »ihre« Assistenten vergeben, durch Seminararbeiten entsprechende Untersuchungen anstellen lassen; das studentische Fußvolk ist für Tests und Propaganda gut.

Ein erster Versuch für den Einsatz von Studenten in der geschilderten Weise ist die in den Sommersemester-Ferien 1970 ausgegebene und in den Juristischen Arbeitsblättern angekündigte Strafrechtsübung von Prof. Blei (FU Berlin). Dazu konnte man sich bei Juradat die einschlägigen Urteile bestellen. Nach einigen Wochen bekam man eine Mustermappe mit Urteilsausschnitten, denen teilweise sogar ein kurzer Hinweis auf den Sachverhalt vorausgestellt war. Darunter befanden sich vier RG-Urteile und vier Urteilsanmerkungen!

$\mathrm{Da}$ diese Werbesendung völlig ohne Thesaurus und ohne Compurer, vielmehr nach herkömmlicher Arbeitsweise (wenn auch in typischer Computer-Druckschrift) zusammengestellt wurde, ist es müßig, näher auf diese, die wahre momentane Arbeitsfähigkeit der Firma bewußt verschleiernde Taktik einzugehen. Es ist leicht vorauszusehen, daß die benutzten Methoden jede wissenschaftliche Forschung mit Hilfe des von Juradat gesammelten Materials verhindern werden. Der Anspruch von Juradat, ein juristisches Forschungs-Zentrum darzustellen, hat außer in der Forschung nach schnellen Profitmöglichkeiten keine sichtbare Manifestation aufzuweisen.

In den kommenden Jahren wird die Rechtswissenschaft, speziell die Rechtsinformatik eine ganze Reihe von Projekten, für die die Hilfe des Computers nötig ist, in Angriff nehmen, so etwa: dialogische Subsumptionshilfen, automatische Subsumption, Sprachstrukturforschung für Thesaurusaufbau; linguistisch-staristische Untersuchungen der Rechtssprache, Sprachprozessor-Programme etc., die sämtlich auf Volltext-Material angewiesen sein dürften. Das Material von $» J u r a-$ dat, mit Hilfe öffentlicher Gelder zusammengestellt, wird wegen der unkontrollierten Informationsfilter und der unzulänglichen Methoden für solche Zwecke untauglich sein. Schon so betrachtet ist jede öffentliche Finanzhilfe an das »Forschungszentrum $\propto$ Juradat eine glatte Fehlinvestition.

Arbeitsgruppe $>E D V$ und Recht\&, Freie Universität Berlin

\section{»Datenverarbeitung und Recht« - Industrialisierung der Justiz?}

Wenn die allgemeine Begeisterung für eine Sache eine gewisse Lautstärke erreicht, besteht meist Grund zur Vorsicht - und zur Analyse des Objekts und der Urheber der Begeisterung. Mir scheint, die Schwärmerei vieler Juristen für die 